\title{
CORRELAÇÃO E REPRODUTIBILIDADE DE TESTES ABDOMINAIS EM MULHERES JOVENS
}

\author{
MS. MAIRA CRISTINA WOLF SCHOENELL
}

Programa de Pós-graduação em Ciências do Movimento Humano, Universidade Federal do Rio Grande do Sul

(Porto Alegre - Rio Grande do Sul - Brasil)

E-mail:mairacws@yahoo.com.br

\section{DR. CARLOS LEANDRO TIGGEMANN}

Programa de Pós-graduação em Ciências do Movimento Humano,

Universidade Federal do Rio Grande do Sul; Faculdade da

Serra Gaúcha; Universidade do Vale do Taquari (UNIVATES)

(Porto Alegre - Rio Grande do Sul - Brasil)

E-mail: cltiggemann@yahoo.com.br

\section{DR. EDUARDO LUSA CADORE}

Programa de Pós-graduação em Ciências do Movimento

Humano, Universidade Federal do Rio Grande do Sul

(Porto Alegre - Rio Grande do Sul - Brasil)

E-mail: edcadore@yahoo.com.br

\section{MS. MARCUS PEIKRISZWILI TARTARUGA}

Departamento de Educação Física, Universidade do

Centro-Oeste de Paraná (UNICENTRO)

(Cascavel - Paraná - Brasil)

E-mail:mtartaruga@hotmail.com

\section{DR. LUIZ FERNANDO MARTINS KRUEL}

Departamento de Desportos, Escola Superior de Educação Física,

Universidade Federal do Rio Grande do Sul

(Porto Alegre - Rio Grande do Sul - Brasil)

E-mail: kruel@esef.ufrgs.br

\section{RESUMO}

O objetivo deste estudo foi relacionar os escores dos testes abdominais de um minuto, de dois minutos com banco, de uma repetição máxima (IRM), de repetições máximas com 70\% de I RM e teste de contração isométrica voluntária máxima (CIVM) e verificar a reprodutibilidades dos mesmos em mulheres jovens. A amostra foi composta de 15 mulheres $(26,22 \pm 4,67$ anos) fisicamente ativas. Os testes foram realizados em dias alternados e randomizados, 
com intervalos mínimos de 48 horas. Após um intervalo de 7 a 10 dias, os testes foram reaplicados. Os resultados sugeriram que os testes abdominais apresentam boa capacidade de reprodutibilidade. Além disso, os testes de um minuto e de dois minutos apresentaram boa relação com o teste de força máxima (IRM), sendo que os testes de CIVM e de repetições máximas a 70\% de IRM não apresentaram correlação com os demais.

PALAVRAS-CHAVE: Força muscular; músculos abdominais; parede abdominal; avaliação física.

\section{INTRODUÇÃO}

Os exercícios abdominais são amplamente utilizados como parte integrante de um programa de exercícios físicos. Um bom nível de força dos músculos abdominais pode reduzir a pressão nos discos intervertebrais lombares, prevenindo doenças degenerativas nos discos (LADEIRA et al., 2005). A força de resistência isométrica da musculatura abdominal tem papel importante na estabilização da coluna, sendo hipoteticamente responsável pelo controle pélvico e lombar e, desta forma, contribuindo para o potencial controle de problemas lombares (KNUDSON, 1999). Além disso, a força da musculatura abdominal assume importância tanto no desempenho atlético quanto na melhoria da aparência física de não atletas (BIRD et al., 2006).

Nas últimas décadas, os exercícios abdominais têm sido o foco de diversas pesquisas. Alguns estudos têm sido realizados buscando avaliar a ativação muscular de diferentes exercícios abdominais (ANDERSSON et al., 1997; STEVENS et al., 2007), com diferentes cargas externas (MORAES et al., 2009), ou então, analisando a ativação muscular em exercícios abdominais realizados em diferentes aparelhos comparando com os tradicionais abdominais realizados no solo (AVEDISIAN et al., 2005; STERNLICHT et al., 2005; BIRD et al., 2006; ESCAMILLA et al., 20 I0; SCHOFFSTALL et al., 20I0). Outros estudos ainda verificaram a influência de diferentes tipos de treinamento na força máxima, na força de resistência e na flexibilidade dos músculos do tronco (SEKENDIZ et al., 2007; STEVENS et al., 2008; VISPUTE et al., 201 I).

A força muscular pode manifestar-se de diferentes formas: máxima, de potência e de resistência, podendo esses componentes ser expressos de forma dinâmica ou isométrica (KNUTTGEN, 1987). Para avaliar essas diferentes manifestações da força, diferentes testes podem ser utilizados, como, por exemplo, os testes de uma repetição máxima (IRM), de contração isométrica voluntária máxima (CIVM), de repetições máximas (RMs), avaliações isocinéticas, entre outros (BROWN; WEIR, 200 I).

Entretanto, para avaliar especificamente a força dos músculos abdominais, historicamente, o teste mais conhecido é o teste de I minuto (sit-up) (KNUDSON, 
1999). Porém, o teste vem sendo criticado nas últimas décadas, por não levar em consideração a ação dos músculos flexores do quadril, como o ílio-psoas, e também, por gerar uma alta pressão entre os discos da coluna vertebral (ANDERSSON et al., 1997; KNUDSON, 1999; KONRAD et al., 200 I). Como alternativa, outros testes abdominais têm sido utilizados visando avaliar a força dos músculos da parede abdominal, como por exemplo, testes abdominais com as pernas apoiadas sobre um banco (KNUDSON; JOHNSTON, 1995).

Poucos autores se propuseram a investigar tais testes e analisar se existem correlações significativas entre os mesmos e os níveis de força máxima ou de resistência, avaliados em dinamômetros de força. Knudson e Johnston (1995) analisaram a validade do teste bench trunk-curl - BTC (abdominal com joelhos curvados e pernas apoiadas sobre um banco) de 2 minutos. Os autores encontraram uma baixa correlação, $r=0,08$ e $r=0,38(p=0,07)$ nos níveis de força e resistência, respectivamente, com o número de repetições do teste BTC.

Em relação à reprodutibilidade dos testes, também são escassas as informações na literatura científica. Knudson e Johnston (1995) testaram a reprodutibilidade do teste BTC de 2 minutos, realizando testes e re-testes em 103 sujeitos, apresentando um alto escore para as mulheres $(r=0,94 ; p=0,00 \mathrm{I})$ e também para os homens $(r=0,88 ; p=0,00 \mathrm{I})$.

Contudo, não foi encontrado na literatura estudos que tenham investigado com maior profundidade dados de correlação entre os tradicionais testes abdominais com outras manifestações da força e testes, assim como, poucos estudos investigaram a reprodutibilidade destes testes. Dessa forma, tem-se o seguinte problema de pesquisa: há uma correlação entre os diferentes testes abdominais e estes têm um nível de reprodutibilidade satisfatório? A hipótese é que os testes que avaliam manifestações diferentes de força não apresentem correlação, mas apresentem reprodutibilidade.

\section{MATERIAIS E MÉTODOS}

A definição do tamanho da amostra foi estabelecida através de cálculo amostral realizado pelo software Pepi 4. I, utilizando como referência os estudos de Knudson e Johnston (1995) e Knudson (200 I ), sendo adotado um nível de significância de 0,05 e um poder de $80 \%$, no qual definiu-se o "n" amostral em I 5 sujeitos. Portanto, a amostra foi composta por 15 sujeitos do sexo feminino com média de idade de 26,22 \pm 4,67 anos e praticantes da modalidade de ginástica Jump. Como critérios de inclusão, adotou-se as seguintes características: fisicamente ativas e sem histórico de dores lombares. Como critérios de exclusão, adotou-se o tempo de prática de ginástica que deveria ser de pelo menos 3 meses e a frequência semanal de prática 
que deveria ser de 2 sessões semanais. Nem todos os sujeitos concluíram todas as avaliações dos testes, justificados por problemas pessoais. Todos leram e assinaram o Termo de Consentimento Livre e Esclarecido, concordando participar do estudo, sendo o estudo aprovado pelo Comitê de Ética em Pesquisa da Universidade de Santa Cruz do Sul sob o parecer n 2267/09.

A avaliação antropométrica foi realizada por um único avaliador, previamente treinado. A caracterização antropométrica foi determinada através dos protocolos estabelecidos por Heyward (2004) quanto à técnica de medida e os pontos anatômicos utilizados. Foram coletadas medidas de massa corporal, estatura e dobras cutâneas (panturrilha, coxa, abdominal, suprailíaca, axilar média, peitoral, triciptal e subescapular). $\bigcirc$ cálculo da densidade corporal foi estimado através da equação de Jackson e Pollock ( 1978) para mulheres. O percentual de gordura (\%G) foi calculado através da fórmula proposta por Siri apud Heyward (2004). A massa corporal magra (MCM) foi determinada a partir da subtração do peso de gordura da massa corporal total. Os resultados da avaliação antropométrica e os dados da caracterização da amostra estão descritos na Tabela I. Anterior à realização dos testes, todos os sujeitos foram submetidos a duas sessões de familiarização com os equipamentos e à execução dos testes envolvidos no experimento.

Tabela I. Média e desvio padrão (DP) das variáveis: idade, peso, índice de massa corporal (IMC), somatório de dobras, gordura corporal e massa corporal magra (MCM)

\begin{tabular}{ll}
\hline Variável $(\mathrm{n}=15)$ & Média \pm DP \\
\hline Idade (anos) & $26,22 \pm 4,67$ \\
Peso $(\mathrm{kg})$ & $62,88 \pm 9,95$ \\
Altura $(\mathrm{m})$ & $1,68 \pm 0,05$ \\
IMC $\left(\mathrm{kg} / \mathrm{m}^{2}\right)$ & $22,15 \pm 3,22$ \\
Somatório de dobras $(\mathrm{mm})$ & $103,93 \pm 34,93$ \\
Gordura Corporal $(\%)$ & $29,49 \pm 5,82$ \\
MCM $(\%)$ & $43,95 \pm 4,91$ \\
\hline
\end{tabular}

protocolo experimental do estudo consistiu na realização de 5 testes abdominais: teste de I minuto, 2 minutos com banco, I RM, RMs e CIVM. Os testes foram agrupados em 3 blocos: Bloco I - Teste de IRM e teste de RMs; Bloco 2 Teste de I minuto e teste de CIVM; e Bloco 3 - Teste de 2 minutos com banco. Respeitou-se um intervalo de 30 minutos entre a realização dos dois testes do bloco I e 2. Cada bloco foi realizado em dias alternados e randomizados entre os sujeitos, com intervalos de no mínimo 48 horas e máximo de 96 horas. Após um intervalo de 7 a 10 dias, todo o protocolo de testes foi reaplicado para a avaliação 
da reprodutibilidade dos mesmos. Desta forma, tem-se a fase de teste I (TI) e a fase de teste 2 (T2). As sessões ocorreram sempre no mesmo horário, entre as 17h e 19h. A aplicação de cada teste seguiu os seguintes procedimentos:

\section{TESTE ABDOMINAL DE I MINUTO}

O teste seguiu o protocolo sugerido por Farinatti (2000), tendo como posição inicial os indivíduos posicionados em decúbito dorsal sobre um colchonete, com os pés fixos e posicionados sobre o solo, estando os calcanhares unidos e a uma distância de 30 a 45 cm do quadril, com os dedos das mãos entrelaçados atrás da cabeça. Os cotovelos deveriam tocar os joelhos na flexão anterior da coluna e cada repetição foi contada no momento em que o sujeito retornasse à posição inicial. Foi contabilizado o máximo de repetições realizadas corretamente no tempo de I minuto.

\section{TESTE ABDOMINAL DE 2 MINUTOS COM BANCO}

Seguindo protocolo de Knudson e Johnston (1995), os indivíduos foram posicionados em decúbito dorsal sobre um colchonete, com flexão de joelhos e quadris, estando as pernas (gastrocnêmios) apoiadas sobre um banco com altura de $46 \mathrm{~cm}$ e os braços cruzados à frente do corpo, tendo as mãos fixadas nos ombros do lado contrário. O movimento de flexão anterior da coluna foi realizado até que os cotovelos encostassem a porção anterior da coxa (quadríceps), sendo que uma repetição foi contabilizada quando o sujeito retornasse à posição inicial. Foi contabilizado o máximo de repetições possíveis no tempo de 2 minutos.

\section{TESTE DE CONTRAÇÃO ISOMÉTRICA VOLUNTÁRIA MÁXIMA (CIVM)}

O teste isométrico foi realizado conforme as orientações de Brown e Weir (200 I). Os indivíduos foram posicionados sentados sobre um banco, com flexão de quadril e de joelhos a $90^{\circ}$, estando os pés fixados. Os indivíduos utilizaram um colete especialmente confeccionado para este estudo, no qual foi fixado o cabo de aço na altura das escápulas, estando o mesmo perpendicular ao tronco do sujeito. Ao cabo estava acoplada uma célula de carga ligada a um conversor análogo digital (A/D) Miotool (ambos da marca Miotec). A célula foi previamente aferida com carga conhecida, a cada novo dia de coleta. No momento da contração isométrica máxima, o tronco encontrava-se flexionado a $45^{\circ}$, sendo que os sujeitos foram orientados a produzirem força de forma "mais rápida e forte possível" (hard and fast) (Sahaly et al., 200 I). Foram realizadas 3 tentativas com duração de 5 segundos cada, respeitando um intervalo de I minuto entre cada tentativa. Durante cada tentativa 
realizada, os sujeitos foram encorajados verbalmente por parte dos pesquisadores para produzirem a máxima força. O sinal de força obtido foi adquirido pelo software Miograph (marca Miotec). Para análise da força isométrica máxima foi verificado o pico de força $(\mathrm{N})$ em cada uma das 3 tentativas, sendo utilizada média das mesmas como resultado final.

\section{TESTES DE IRM E RMS}

Os testes de I RM e RMs foram realizados em um aparelho Flexor de Coluna, modelo Fusion da marca Pórtico, com resolução de $5 \mathrm{~kg}$ e capacidade de $70 \mathrm{~kg}$. Cargas adicionais de $1 \mathrm{~kg}$ e $2 \mathrm{~kg}$ foram utilizadas para melhor resolução dos testes. Segundo recomendações de Brown e Weir (200 I), o teste de IRM foi realizado através do procedimento de tentativa e erro, no qual cargas progressivamente maiores foram utilizadas até que apenas uma repetição fosse possível de ser executada, sendo que nenhum sujeito necessitou mais de 5 tentativas. Intervalo de 3 a 5 minutos foi respeitado entre as tentativas. A amplitude do movimento foi determinada a partir do ponto zero (com o tronco na posição vertical) até o mesmo atingir $45^{\circ}$ (KNUDSON, 1999).

No teste de RMs, utilizando uma carga correspondente a 70\% do teste de I RM, foi contabilizado o número máximo de repetições realizadas de forma tecnicamente correta (DOURIS, 2006). A velocidade de realização do movimento de flexão foi monitorada de forma visual, sendo indicada a utilização de uma velocidade moderada $(\approx 1,5$ segundos cada fase - concêntrica e excêntrica). A amplitude foi a mesma adotada no teste de IRM.

\section{ANÁLISE ESTATÍSTICA}

Os dados foram considerados normais através do teste de Shapiro-Wilk e estão apresentados através de médias e desvio padrão. A correlação entre os diferentes testes foi feita através do teste de Correlação de Pearson, a reprodutibilidade testada através do teste de correlação intraclasse (ICC), sendo que para a comparação entre TI e T2 dos testes, o Teste-t pareado foi utilizado. O nível de significância adotado foi de $5 \%(p<0,05)$, sendo que todos os procedimentos foram realizados pelo programa SPSS (v. 15.0).

\section{DISCUSSÃO}

Os dados descritivos das etapas TI e T2, bem como a comparação entre os mesmos, estão apresentados na Tabela 2. Diferenças significativas foram encontradas nos testes de IRM e RMs ( $p<0,05)$. Valores significativos de ICC (TI e T2) foram encontrados nos testes de $I \min (r=0,82 ; p=0,00 I), 2 \min (r=0,9 \mid$; 
$p=0,001)$, IRM $(r=0,90 ; p=0,001)$ e de CIVM $(r=0,63 ; p=0,004) .0$ teste de RMs não apresentou valor de reprodutibilidade significativo $(r=0,40$; $p=0,060)$.

Tabela 2. Média e desvio padrão (DP) dos testes de I minuto (I min), 2 minutos (2 min), I repetição máxima (I RM), repetições máximas (RMs) e contração isométrica voluntária máxima (CIVM) da etapa de os testes ( $\mathrm{TI}$ ) e re-testes (T2), com o respectivo valor de significância (p); rep = repetições;

$\mathrm{kg}$ = quilogramas; $\mathrm{N}=$ newtons

\begin{tabular}{|c|c|c|c|}
\hline \multirow{2}{*}{ Testes / Unidade/ (n) } & \multicolumn{2}{|l|}{ Média \pm DP } & \multirow[b]{2}{*}{$p$} \\
\hline & $\mathrm{TI}$ & T2 & \\
\hline $1 \mathrm{~min} / \mathrm{rep} /(\mathrm{n}=16)$ & $26,19 \pm 4,51$ & $27,56 \pm 5,45$ & 0,083 \\
\hline $2 \mathrm{~min} / \mathrm{rep} /(\mathrm{n}=17)$ & $41,82 \pm 13,42$ & $43,88 \pm 13,48$ & 0,142 \\
\hline I RM / kg / $(n=15)$ & $46,53 \pm 13,30$ & $50,87 \pm 12,29$ & $0,008 *$ \\
\hline $\mathrm{RMs} / \mathrm{rep} /(\mathrm{n}=15)$ & $14,80 \pm 6,24$ & $9,80 \pm 2,54$ & $0,002 *$ \\
\hline $\mathrm{CIVM} / \mathrm{N} /(\mathrm{n}=15)$ & $430,93 \pm|| 0,0 \mid$ & $420,31 \pm 90,76$ & 0,493 \\
\hline
\end{tabular}

* Nível de significância $p<0,05$.

Os principais achados deste estudo sugerem haver moderados a altos valores de reprodutibilidade dos diferentes testes abdominais, com exceção do teste de RMs.

Foram encontradas diferenças significativas nos testes de IRM e RMs nas etapas TI e T2. Possivelmente isso possa ser explicado pelo fato de alguns sujeitos não terem conseguido realizar seu máximo esforço no teste de IRM, e consequentemente, possibilitando a realização de muitas repetições no teste de RMs, na etapa TI . Já na etapa T2, possivelmente através de uma maior familiarização com o teste, causado pelo efeito da aprendizagem da etapa TI, os indivíduos podem ter alcançado maiores cargas máximas no teste de IRM, e consequentemente, reduzindo o número de repetições no teste de RMs (CRONIN; HENDERSON, 2004; DIAS et al., 2005). Salientamos que os sujeitos foram familiarizados com o equipamento e o movimento utilizado, mas não com as altas cargas especificamente. Assim, possivelmente, o fato dos sujeitos da pesquisa serem praticantes da modalidade jump, não estando os mesmos habituados ao treinamento com cargas (CRONIN; HENDERSON, 2004) próximas à força máxima, dificulte aos mesmos a produção de força com este tipo de carga. Este argumento pode também ser corroborado pela inexistência de diferenças significativas entre as etapas TI e T2 dos testes de I min, 2 min e CIVM, ou seja, testes com uma maior familiarização por parte da amostra e facilidade de execução é verificado, contribuindo para a aquisição de resultados mais similares.

Em relação à reprodutibilidade dos testes, foram encontrados índices fortes e significativos de reprodutibilidade nos testes de I minuto, 2 minutos e IRM, índice moderado no teste de CIVM e índice baixo e não significativo do teste de 
RMs. Esses resultados concordam com os resultados do Teste-t, afinal os testes de I minuto, 2 minutos e CIVM apresentaram comportamento e resultados similares. teste de IRM apresentou médias diferentes nas duas etapas ( $T$ I e T2), porém demonstrou um comportamento similar, ou seja, com aumento do valor da carga na segunda etapa, gerando, desta forma, valores significativos de ICC. Já no teste de RMs, possivelmente a grande variabilidade dos dados encontrados, principalmente na primeira etapa (TI de 7 a 25 RMs; T2 de 7 a 14 RMs), possa ter contribuído pela não existência de correlação entre as duas etapas. Outros estudos também verificaram bons valores de reprodutibilidade entre os diferentes testes. Knudson e Johnston (1995) verificaram a reprodutibilidade do teste de 2 minutos aplicados a mulheres, encontrando correlações altas e significativas $(r=0,94 ; p<0,05)$. Em relação ao teste de I RM, (TIGGEMANN et al., 20 I 0) verificaram a reprodutibilidade nos exercícios de supino e pressão de pernas, encontrando correlações altas e significativas em diferentes situações, mesmo apresentando diferenças significativas entre os momentos de coleta ( $r=0,922$ a 0,997; $p<0,05)$.

Os resultados de correlação entre os testes estão apresentados na Tabela $3(\mathrm{TI})$ e 4 (T2). Correlações moderadas e significativas foram encontradas apenas entre os testes de I minuto e 2 minutos, e I minuto e IRM na etapa TI, e além destas, entre os testes de 2 minutos e IRM na etapa T2.

Tabela 3. Valores de correlação entre os testes de I minuto (I min), 2 minutos (2 min), I repetição máxima (I RM), repetições máximas (RMs) e contração isométrica voluntária máxima (CIVM) da etapa I de testes $(\mathrm{TI})$

\begin{tabular}{llllll}
\hline & I min & $2 \min$ & | RM & RMs & CIVM \\
\hline I $\min (n=16)$ & 1,000 & $0,585 *$ & $0,730 *$ & $-0,016$ & $-0,094$ \\
$2 \min (n=17)$ & & 1,000 & 0,408 & $-0,399$ & $-0,022$ \\
$\mid \operatorname{RM}(n=15)$ & & & 1,000 & $-0,090$ & $0,07 \mid$ \\
$\operatorname{RMs}(n=\mid 5)$ & & & & 1,000 & $-0,083$ \\
CIVM $(n=15)$ & & & & & 1,000 \\
\hline
\end{tabular}

* Nível de significância $p<0,05$.

Tabela 4. Valores de correlação entre os testes de I minuto (I min), 2 minutos (2 min), I repetição máxima (I RM), repetições máximas (RMs) e contração isométrica voluntária máxima (CIVM) da etapa 2 de retestes (T2)

\begin{tabular}{llllll}
\hline Testes & I min & 2 min & I RM & RMs & CIVM \\
\hline I $\min (n=16)$ & 1,000 & $0,633^{*}$ & $0,724^{*}$ & $-0,003$ & 0,088 \\
$2 \min (n=17)$ & & 1,000 & $0,654^{*}$ & $-0,170$ & 0,210 \\
I RM $(n=15)$ & & & 1,000 & $-0,070$ & 0,047 \\
RMs $(n=15)$ & & & 1,000 & $-0,152$ \\
CIVM $(n=15)$ & & & & 1,000 \\
\hline
\end{tabular}

* Nível de significância $p<0,05$ 
No que diz respeito às correlações entre os 5 testes abdominais, somente em alguns pares de comparação, correlações significativas foram encontradas. Em relação às correlações encontradas entre os testes de I minuto e 2 minutos, a mesma pode ser explicada em função de ambos os testes terem movimentos similares, embora ocorra uma flexão anterior da coluna mais acentuada no teste de I min. Knudson e Johnston (1998) analisaram o teste de banco em diferentes tempos de 60s, 90s e 120s, encontrando correlações altas e significativas entre os três tempos do teste $(0,88$ a 0,$91 ; p<0,01)$.

Também correlações altas e significativas foram encontradas entre o teste de IRM e os testes de I minuto e 2 minutos. Historicamente, o teste de I minuto é utilizado para verificar a força de resistência dos músculos da parede abdominal, bem como, o teste de I RM se propondo a avaliar a força máxima dos sujeitos. Mas, sabendo que nos testes de I minuto e 2 minutos há limitação do tempo na execução das repetições, exige dos sujeitos a realização das repetições em máxima velocidade. Desse modo, hipotetizamos que, provavelmente, o teste de I minuto não represente uma força puramente de resistência, mas uma mescla de força de potência e força máxima. Outra possível explicação diz respeito a uma melhor economia neuromuscular dos sujeitos com maiores valores de força máxima, ou seja, sujeito com maiores valores de I RM teriam maior capacidade de realizar maior número de repetições nos testes de I minuto e 2 minutos, pelo fato de necessitarem menor quantidade de unidades motoras para a realização do esforço não máximo (CADORE et al., 20 l 0). Poucos estudos foram encontrados relacionando diferentes manifestações da força na musculatura abdominal. Knudson e Johnston (1995), quando relacionaram diferentes testes abdominais, encontraram baixa correlação $(r=0,38 ; p=0,07)$ entre o teste de 2 minutos e a força de resistência, e uma correlação ainda menor ( $r=0,08 ; p=0,07$ ) entre o teste de 2 minutos e a força máxima (pico de torque isocinético). Segundo os autores, essas baixas correlações poderiam ser explicadas em consequência de dois fatores: a diferença de posição do corpo nos testes e a diferença de velocidade de execução. Assim, no teste em equipamento isocinético o sujeito parte da posição sentada e realiza o teste em velocidade predeterminada, enquanto que no teste de 2 minutos, o sujeito inicia o teste na posição deitada e realiza as flexões de tronco na sua máxima velocidade durante o mesmo.

Em relação à inexistência de correlação entre os testes de IRM e o teste de RMs, a mesma pode ter sido influenciada pelo fato dos sujeitos não estarem completamente familiarizados com o teste máximo, conforme visto anteriormente, e ainda, pela falta de um controle mais rígido na velocidade de execução das repetições no teste de RMs. Fato este que pode ser uma limitação do presente estudo. Embora que, na fase de familiarização, os sujeitos tenham sido instruídos a 
realizarem os movimentos de forma lenta e contínua, usando aproximadamente 3 segundos para cada repetição, sendo I,5 segundos para cada fase do movimento (concêntrica e excêntrica), possivelmente durante o teste, este padrão possa não ter sido respeitado por todos os sujeitos. Assim, possivelmente os sujeitos que tenham utilizado velocidades maiores, tenham conseguido realizar uma maior quantidade de repetições, (SAKAMOTO; SINCLAIR 2006) gerando assim uma grande variabilidade das respostas entre os mesmos, comprometendo os dados de correlação. Contudo, importante observar que, na segunda etapa, o número de RMs variou entre 7 e I 5 RMs entre os sujeitos, sendo este valor similar a outros estudos. Douris (2006) encontram valores que variaram entre 7 a I 4 RMs no exercício extensão de joelhos com $70 \%$ de I RM e, embora os autores tenham encontrado uma baixa correlação $(r=-0,48 ; p=0,04)$ entre o número de RMs e o tipo de fibra, os mesmos justificam que este fator possa determinar a variabilidade entre os sujeitos, ou seja, sujeitos com maior percentual de fibras do tipo I conseguiriam realizar maior quantidade de RMs, fator este que também poderia auxiliar no entendimento de nossos achados.

No presente estudo o teste de CIVM não apresentou correlação com nenhum outro teste dinâmico, sendo que estes resultados confirmam uma possível controvérsia encontrada na literatura. Afinal, autores se propuseram a comparar a força dinâmica e a força isométrica em atividades de saltos e corridas, mas encontraram resultados diferentes. Enquanto alguns autores mostram que a força isométrica se relaciona com a força dinâmica (VIITASALO et al., I98I ; VIITASALO, 1984; JARIC et al., 1989), outros relatam que as mesmas não possuem nenhuma relação (MERO et al., 198I; YOUNG, 1993; WILSON; MURPHY, 1995). Haff et al. ( 1 997) analisaram essa discordância na literatura, salientando que movimentos com alta potência tendem a gerar mais força isométrica, enquanto que atividades de resistência utilizariam pouca força isométrica. Portanto, mais estudos ainda são necessários para relacionar a força dinâmica com a força isométrica.

Baseando-se nestes resultados, pode-se concluir que existem fortes relações entre o teste de força máxima (I RM) e os testes de I minuto e 2 minutos, bem como entre os testes de I minuto e 2 minutos em mulheres jovens. A força isométrica máxima não apresentou relação com os testes de força dinâmica, sugerindo possivelmente um padrão diferenciado da força muscular entre estas duas manifestações da força. Em relação à reprodutibilidade, verificamos que testes abdominais podem ser bem reproduzíveis nesta população, sendo que uma atenção especial deve ser dada aos testes de I RM e RMs, principalmente quanto a uma correta familiarização e controle da velocidade. 


\section{Correlation and Reproducibility Testing Abdominals in Young Women}

ABSTRACT: The purpose of this study was to investigate possible correlations between the values of I minute abdominal tests, 2 minutes with bench, a repetition maximum, a repetition maximum with $70 \%$ of I RM and maximum voluntary isometric contraction test and then, to verify the reproducibility of them in young women. The study was composed by 15 trained women (26.22 \pm 4.67 ages). The tests were randomly performed in different days with minimum of 48 hours of interval between collections. After of 7- 10 days interval, the tests were performed again. The present results suggested it is possible to conclude that abdominal tests present a good capacity of reproducibility. Besides this, the I min and $2 \mathrm{~min}$ tests have presented a good relation with the maximum strength test (IRM), however the maximum voluntary isometric contraction and repetition maximum with $70 \%$ of I RM tests did not presented any correlation with the other tests.

KEYWORDS: Muscle strength; abdominal muscle; abdominal wall; physical evaluation.

\section{Correlación y Reproducibilidad de Diferentes Tests Abdominales en mujeres jóvenes}

RESUMEN: El objetivo de esta investigación fue relacionar la puntuación de los resultados de los siguientes tests abdominales: abdominal en un minuto, abdominal en dos minutos en la banca, una repetición máxima (IRM), repeticiones máximas a 70\% de IRM y test de contracción isométrica voluntaria máxima (CIVM); y evaluar la reproducibilidad de los mismos en mujeres jóvenes. La muestra estuvo constituída por 15 mujeres (26,22 \pm 4,67 años) físicamente activas. Los tests se realizaron en días distintos y al azar, con intervalos de 48 horas. Después de 7-10 días los tests fueron aplicados nuevamente. Los resultados sugieren que los ejercicios abdominales muestran una buena capacidad de reproducibilidad. Además, los tests de un minuto y dos minutos mostraron buena relación con la prueba de I RM, pero los testes de CIVM y repeticiones máximas a 70\% de I RM no mostraron ninguna correlación con los demás.

PALABRAS CLAVE: Fuerza muscular; músculos abdominales; pared abdominal; evaluación física.

\section{REFERÊNCIAS}

ANDERSSON, E. et al. Abdominal and hip flexor muscle activation during various training exercises. European Journal of Applied Physiology and Occupational Physiology, Berlin, n.75, v.2 p.|15-123, mar. 1997.

AVEDISIAN, L. et al. Abdominal strengthening using the abvice machine as measured by surface electromyographic activation levels. Journal of Strength and Conditioning Research, Colorado Springs, n. 19, v. 3, p. 709-712, aug. 2005.

BIRD, M.; FLETCHER, K. M.; KOCH, A. J. Electromyographic comparison of the ab-slide and crunch exercises. Journal of Strength and Conditioning Research, Colorado Springs, n. 20, v. 2, p. 436-440, may 2006. 
BROWN, L. E.; WEIR, J. P. Asep procedures recommendation I: accurate assesment of muscular strenght and power. Journal of Exercise Physiology on line, Duluth, n. 4, v. 3, p. I-2I, aug. 200I.

CADORE, E. et al. Neuromuscular economy, strength and endurance in healthy elderly men. Journal of Strength and Conditioning Research, Colorado Springs, n. 25, v. 4, p. $997-$ 1003, apr. 201।.

CRONIN, J. B.; HENDERSON, M. E. Maximal strength and power assessment in novice weight trainers. Journal of Strength and Conditioning Research, Colorado Springs, n. I8, v. I, p. 48-52, feb. 2004.

DIAS, R. M. R. et al. Influência do processo de familiarização para avaliação da força muscular em testes de I-RM. Revista Brasileira de Medicina do Esporte, São Paulo, v. I, n. I I, p. 34-42, jan./fev. 2005.

DOURIS, P. C. et al. The relationship between maximal repetition performance and muscle fiber type as estimated by noninvasive technique in the quadriceps of untrained women. Journal of Strength and Conditioning Research, Colorado Springs, n. 20, v. 3, p. 699-703, aug. 2006.

ESCAMILLA, R. F. et al. Core muscle activation during swiss ball and traditional abdominal exercises. Journal of Orthopaedic and Sports Physical Therapy, Alexandria, n. 40, v. 5, p. 265 276, may 2010.

FARINATTI, P. D. T. V. Fisiologia e avaliação funcional. 4. ed. Rio de Janeiro: Sprint, 2000. v. I. p. 302.

HAFF, G. et al., Force-time dependent characteristics of dynamic and isometric muscle actions. Journal of Strength and Conditioning Research, Colorado Springs, n. I I, v. 4, p. 269272, nov. 1997.

HEYWARD, V. H. Avaliação física e prescrição de exercício: técnicas avançadas. Porto Alegre: Artmed, 2004. p. 319.

JARIC, S.; RISTAMOVIC, D.; CORCOS, D. M. The relationship between muscle kinematic parameters and kinematic variables in a complex movement. European Journal of Applied Physiology and Occupational Physiology, Berlin, n. 59, v. 5, p. 370-376, dec. 1989.

KNUDSON, D. Issues in abdominal fitness: testing and technique. Journal of Physical Education, Recreation and Dance, Reston, n. 70, v. 3, mar. 1999.

KNUDSON, D. The validity of recent curl-up tests in young adults. Journal of Strength and Conditioning Research, Lincoln, n. I5, v. I, p. 81-85, feb. 2001.

KNUDSON, D.; JOHNSTON, D. Validity and reliability of a bench trunk-curl test of abdominal endurance. Journal of Strength and Conditioning Research, Colorado Springs, n. 9, v. 3, p. 165-169, aug. 1995. 
KNUDSON, D.; JOHNSTON, D. Analysis of three test durations of the bench trunk-curl." Journal of Strength and Conditioning Research, Colorado Springs, n. I2, v. 3, p. I50- I 5 I, aug. 1998.

KNUTTGEN, N. H. E. K. Terminology e measurement in exercise performance. Journal of Applied Sport Science Research, Lincoln n. I, v. I, p. I-10, feb. 1987.

KONRAD, P. et al. Neuromuscular Evaluation of Trunk-Training Exercises. Journal of Athletic Training, Dallas, v. 36, n. 2, p. 109-1 I8, apr./jun. 2001.

LADEIRA, C. E. et al. Validation of an abdominal muscle strength test with dynamometry. Journal of Strength and Conditioning Research, Colorado Springs, n. 19, v. 4, p. 925-930, nov. 2005.

MERO, A. et al. Relationship between maximal running velocity, muscle fiber characteristies, force production and force relaxation of sprinters. Scandinavian Journal of Sport Science, Helsinki, n. 3, p. 16-22, mar. 1981.

MORAES, A. C. et al. EMG activation of abdominal muscles in the crunch exercise performed with different external loads. Physical Therapy in Sport, Edinburgh, n. 10, v. 2, p. 57-62, jan. 2009.

SAHALY, R.; H. VANDEWALLE, et al. Maximal voluntary force and rate of force development in humans - Importance of instruction. European Journal of Applied Physiology and Occupational Physiology, Berlin, v. 3/4, n. 85, p. 345-350, aug. 2001.

SAKAMOTO, A.; SINCLAIR, P. J. Effect of movement velocity on the relationship between training load and the number of repetitions of bench press. Journal of Strength and Conditioning Research, Colorado Springs, n. 20, v.3, p. 523-527, aug. 2006.

SCHOFFSTALL, J. E.; TITCOMB, D. A.; KILBOURNE, B. F., Electromyographic response of the abdominal musculature to varying abdominal exercises. Journal of Strength and Conditioning Research, Colorado Springs, v. 12, n. 24, p. 3422-3426, dec. 2010.

SEKENDIZ, B.; ALTUN, O. et al. Effects of Pilates exercise on trunk strength, endurance and flexibility in sedentary adult females. Journal of Bodywork and Movement Therapies, New York, n. II, v.4, p. 318-326, oct. 2007.

STERNLICHT, E. et al. Electromyographical analysis and comparison of selected abdominal training devices with a traditional crunch. Journal of Strength and Conditioning Research, Colorado Springs, n. 19, v. I, p. 157-162, feb. 2005.

STEVENS, V. K.; P. L. COOREVITS, et al. The influence of specific training on trunk muscle recruitment patterns in healthy subjects during stabilization exercises. Manual Therapy, Edinburgh, n. 12, v. 3, p. 27I-279, aug. 2007.

STEVENS, V. K. et al. The effect of increasing resistance on trunk muscle activity during extension and flexion exercises on training devices. Journal of Electromyography and Kinesiology, New York, n. 18, v. 3, p. 434-445, june 2008. 
TIGGEMANN, C. L. et al. Perceived exertion in different strength exercise loads in sedentary, active, and trained adults. Journal of Strength and Conditioning Research, Colorado Springs, v. 8, n. 24, p. 2032-204I, aug. 2010.

VISPUTE, S. S. et al. The effect of abdominal exercise on abdominal fat. Journal of Strength and Conditioning Research, Colorado Springs, v. 9, n. 25, p. 2559-2564, sept. 201 I.

VIITASALO J.T. Seasonal fluctuation of force production in higt jumpers. Canadian Journal of Applied Sport Sciences, Vanier, v. 4, n. 9, p. 209-213, dec. 1984.

VIITASALO, J. T.; HAKKINEM K.; KOMI, P. V. Isometric and dinamic force production and muscle fibre composition in man. Journal Human Movement Study, Edinburgh, n. 7, 199-209, 1981.

WILSON, G. J. ; MURPHY, A.J. The efficacy of isokinetic, isometric and vertical jump test in exercise science. Australian Journal of Science Medicine Sports, Pennant Hills, v. I, n. 27 , p. 62-66, mar. 1995.

YOUNG, B. W. E. B. The effect of voluntary effort to influence speed of contraction on strength, muscular power, and hypertrophy development. Journal of Strength and Conditioning Research, Colorado Springs, v. 3, n. 7, p. 172-178, aug. 1993.

Recebido em: 18 out. 201 I

Aprovado em: 27 maio. 2012

Endereço para correspondência:

Maira Cristina Wolf Schoenell

Rua 2 Norte, 1049

Bairro Centro Administrativo

Teutônia-RS

CEP: 95890-000 